\title{
SQUARE SUMMABILITY OF VARIATIONS AND CONVERGENCE OF THE TRANSFER OPERATOR
}

\author{
ANDERS JOHANSSON AND ANDERS ÖBERG
}

\begin{abstract}
In this paper we study the one-sided shift operator on a state space defined by a finite alphabet. Using a scheme developed by Walters [13, we prove that the sequence of iterates of the transfer operator converges under square summability of variations of the $g$-function, a condition which gave uniqueness of a $g$-measure in [7. We also prove uniqueness of so-called $G$-measures, introduced by Brown and Dooley [2], under square summability of variations.
\end{abstract}

\section{INTRODUCTION}

We consider the left one-sided shift map $T$ on the state space $X^{+}=S^{\mathbb{Z}_{+}}$, where $S$ is a finite set. Thus $T$ acts on elements $x$ of $X^{+}, x=\left(x_{0}, x_{1}, x_{2}, \ldots\right)$, in the following way (each $x_{i}$ belongs to $S$ ):

$$
T\left(x_{0}, x_{1}, x_{2}, \ldots\right)=\left(x_{1}, x_{2}, \ldots\right) .
$$

A $g$-measure associated to a continuous function $g: S^{\mathbb{Z}_{+}} \rightarrow[0,1]$ is a $T$ invariant measure $\mu$ in the space of Borel probability measures $\mathcal{P} X^{+}$, such that $g=d \mu / d \mu \circ T$. Such a function is referred to as a $g$-function and satisfies $\sum_{y \in T^{-1} x} g(y)=1$, for all $x \in X^{+}$, and thus can be viewed as a probability transition function for the local inverses of $T$. Keane [6] introduced the concept of $g$-measures into Ergodic Theory.

Existence of at least one $g$-measure is automatic if $S$ is finite and if $g>0$, since then $S^{\mathbb{Z}_{+}}$is compact, and the continuity of $g$ implies that inf $g>0$. If $S$ is countably infinite, then existence is no longer automatic, but a weak sufficient condition was given in [8].

Recent results concerning necessary conditions for uniqueness of a $g$-measure have been given by Berger, Hoffman and Sidoravicius [1] and also by Hulse [5]

Necessary and sufficient conditions are sometimes, but not always, centered around conditions on the variations of the $g$-function on small parts of its

2000 Mathematics Subject Classification. 37A05 (28D05 37A30 37A60).

Key words and phrases. Transfer operator, g-function, $g$-measure. 
domain. For $f: X^{+} \rightarrow \mathbb{R}$, the variation is defined as

$$
\operatorname{var}_{n} f=\sup _{x \sim_{n} y}|f(x)-f(y)|,
$$

where $x \sim_{n} y$ if $x, y \in X^{+}$coincide in the first $n$ coordinates.

Walters [12] proved uniqueness and rates of convergence under summable variations of $g$-functions, or rather their logarithms, $\log g$, which amounts to the same thing, if inf $g>0$.

When $S$ is finite, a sufficient condition for uniqueness of a $g$-measure, square summability of variations of the $g$-function, was found by the present authors [7] and this result was extended to countable state shifts in Johansson, Öberg and Pollicott [8].

Square summability of variations of a $g$-function then means that

$$
\sum_{n=1}^{\infty}\left(\operatorname{var}_{n} f\right)^{2}<\infty
$$

In this paper we prove convergence of the iterates of the transfer (transition) operator, which is defined by

$$
\mathcal{L} f(x)=\sum_{y \in T^{-1} x} g(y) f(y),
$$

for continuous functions $f$ on $X^{+}$.

More specifically, we prove that with finitely many symbols in $S$ and under square summability of variations of a strictly positive $g$-function, we have $\mathcal{L}^{n} f(x) \rightarrow \int f d \mu$, uniformly in $x$.

To accomplish this, we use a theory developed by Walters in [13] to study the sequence of iterates of an operator $P$, which is defined as

$$
P^{n} f(x)=\left(\mathcal{L}^{n} f\right)\left(T^{n} x\right),
$$

for $n \geq 1$.

Walters introduces an adjoint sequence of operators $P^{(n)^{*}}: \mathcal{P} X^{+} \rightarrow \mathcal{P} X^{+}$, $n \geq 1$. Define $K_{g}^{n}=\left\{\nu \in \mathcal{P} X^{+} \mid P^{(n)^{*}} \nu=\nu\right\}$. Then (Theorem 2.1(v) of [13]) implies that $K_{g}^{n}=P^{(n)^{*}} \mathcal{P} X^{+}$and that $K_{g}^{1} \supset K_{g}^{2} \supset \ldots$ Walters defined $K_{g}=\bigcap_{n=1}^{\infty} K_{g}^{n}$, which is the set of $G$-measures in the terminology of Brown and Dooley [2]. See also Fan [4] and Dooley and Stenflo [3] for some recent contributions to uniqueness of $G$-measures. The motivation for studying $G$ measures has to some extent been to obtain a more general framework for studying Riesz products, see [4, for example. 
Our strategy is now to prove that there exists a unique measure in $K_{g}$ (it is trivial that any $g$-measure must be an element of $K_{g}$ ). Then we use Theorem 2.9 of [13] to conclude that $\mathcal{L}^{n} f(x) \rightarrow \int f d \mu$, where $\mu$ is the unique element of $K_{g}$. This follows by a compactness argument, so in order to prove the convergence of $\mathcal{L}^{n} f$, we have assumed that the symbol space $S$ is finite.

We need to define a probability measure, which is a Markov chain on $X^{+}$, which we call a $g$-chain, in order to reverse the dynamics in the absence of stationarity; it is not known if (when) the measures in $K_{g}$ are invariant under $T$.

To conclude the proof, we use the same method as in [7] to prove that two extremal measures in $K_{g}$ must be absolutely continuous, and hence that there exists only one such measure. This method was developed further in [8] to cover the case of countable state shifts (thus improving on the results of [7]) for $g$-measures, using more sophisticated methods to prove absolute continuity of measures. These methods had appeared much earlier in the literature by Shiryaev and co-authors, see e.g. the survey by Shiryaev [11].

We end the paper with two open questions.

\section{Results AND PROOFS}

Theorem 2.1. Assume we have a finite symbol set $S$ and let $g>0$ satisfy

$$
\sum_{n=1}^{\infty}\left(\operatorname{var}_{n} \log g\right)^{2} \asymp \sum_{n=1}^{\infty}\left(\operatorname{var}_{n} g\right)^{2}<\infty
$$

and let $\mu$ be the corresponding unique g-measure. Then $K_{g}=\{\mu\}$, or equivalently: there exists a unique $G$-measure, which is the same as the unique g-measure.

The following theorem is in view of [13] a corollary of Theorem 2.1. It uses the connection of Theorem 2.9 of [13] and we copy part of its proof for the convenience of the reader, since it is here explained why we have assumed that we have finitely many symbols (one has to have a compact state space, or at least some tightness property) so that there exists a certain convergent subsequence for a sequence of measures.

Theorem 2.2. Assume we have a finite symbol set $S$ and let $g>0$ be a $g$ function with square summable variation and let $\mu$ be the corresponding unique g-measure. Then $\sup _{x}\left|\mathcal{L}^{n} f(x)-\int f d \mu\right| \rightarrow 0$.

Proof. (Borrowed from Theorem 2.9 of [13.) Under the same assumptions, Theorem 2.1 gave us a unique measure in $K_{g}$. From this it follows that 
$\sup _{x}\left|P^{n} f(x)-\int f d \mu\right| \rightarrow 0$, since otherwise, there exists a continuous function $f$ and an $\epsilon$ and sequences $\left\{n_{k}\right\}$ and $\left\{x_{k}\right\}$ such that

$$
\left|\int f d\left(P^{\left(n_{k}\right)^{*}} \delta_{x_{k}}\right)-\int f d \mu\right| \geq \epsilon \forall k \geq 1 .
$$

We can now, due to compactness, pick a subsequence $\left\{k_{j}\right\}$ of $\{k\}$ such that the sequence of measures $\left\{P^{\left(n_{k_{j}}\right)^{*}} \delta_{x_{k_{j}}}\right\}$ converges to $\nu \in \mathcal{P} X^{+}$. But then since $K_{g}^{(n)}=P^{(n)^{*}} \mathcal{P} X^{+}$, we also have $\nu \in K_{g}$, a contradiction. Since $P^{(n)} f(x)=$ $\left(\mathcal{L}^{n} f\right)\left(T^{n} x\right)$, we also have $\sup _{x}\left|\mathcal{L}^{n} f(x)-\int f d \mu\right| \rightarrow 0$.

2.1. Proof of Theorem 2.1. We begin with some preliminary terminology; see [8] for a more thorough exposition. For a pair of probability measures $\nu, \tilde{\nu} \in$ $\mathcal{P} X$ and some filtration, $\left\{\mathcal{F}_{n}\right\}$, let $Z_{n}(x)=Z_{n}\left(x ; \tilde{\nu}, \nu, \mathcal{F}_{n}\right)$ be the likelihood ratio martingale

$$
Z_{n}\left(x ; \tilde{\nu}, \nu,\left\{\mathcal{F}_{n}\right\}\right)=\frac{\left.d \tilde{\nu}\right|_{\mathcal{F}_{n}}}{\left.d \nu\right|_{\mathcal{F}_{n}}}(x)
$$

on $\mathcal{F}_{n}$, where we assume that $\tilde{\nu}$ and $\nu$ are locally absolute continuous on $\mathcal{F}_{n}$ (see [8]).

Now, let $X=S^{\mathbb{Z}}$ and $X^{+}=S^{\mathbb{Z}_{+}}$and extend the one-sided shift $T$ on $X^{+}$to a two-sided shift on $X$. For $a, b \in \mathbb{Z}$ let $\Pi_{a, b}(x)$ be the mapping taking $x \in X$ to $\left(x_{a}, x_{a+1}, \ldots, x_{b}\right) \in S^{b-a}$ and define the natural projection $\Pi_{+}: X \rightarrow X^{+}$ taking bi-infinite sequences $x$ in $X$ to one-sided ones in $X^{+}$by $\Pi_{+}\left(\left(x_{i}\right)_{i=-\infty}^{i=\infty}\right)=$ $\left(x_{0}, x_{1}, \ldots\right)$. Let also $\mathcal{F}_{a}^{b}$ be the algebra generated by $\Pi_{a, b}$ and $\mathcal{F}_{n}^{-}:=\mathcal{F}_{-n}^{-1}$ (the "forward" algebra) and $\mathcal{F}_{n}^{+}:=\mathcal{F}_{0}^{n-1}$ (the "backward" algebra). A cylinder set is a set of the form $[x]_{a}^{b}=\Pi_{a, b}^{-1} \Pi_{a, b}(x)$.

We will now define a certain Markov chain, a $g$-chain in order to be able to go forward in time, since we may not assume that the measures in $K_{g}$ defined by Walters are invariant under $T$.

Definition 1. A $g$-chain on $X=S^{\mathbb{Z}}$ is a probability measure $\nu \in \mathcal{P} X$ such that, for all $n$ in $\mathbb{Z}$,

$$
\nu\left(x_{n} \mid x_{n+1}, x_{n+2}, \ldots\right)=g\left(x_{n}, x_{n+1}, x_{n+2}, \ldots\right) .
$$

A forward $g$-chain is a probability measure $\nu$ on $X$ satisfying (2.1) for $n \leq$ -1 .

For a $g$-chain $\nu$, the distribution under $\nu$ of the process $x^{(t)} \in X^{+}, t \in \mathbb{Z}$, is defined by

$$
x^{(t)}:=\Pi_{+}\left(T^{-t} x\right)=\left(x_{-t}, x_{-t+1}, \ldots\right),
$$


is that of a Markov chain such that the transition probabilities are given by $g$ (and the transition operator of the chain is $\mathcal{L}$ ). That is, for all $t \in \mathbb{Z}$,

$$
\nu\left(x^{(t)} \mid x^{(t-1)}\right)=g\left(x^{(t)}\right) .
$$

The same holds for $t \geq 1$ in the case of a forward $g$-chain $\nu$.

Lemma 2.3. A probability measure $\nu \in \mathcal{P} X$ is a g-chain only if $\nu \circ \Pi_{+}^{-1} \in \mathcal{P} X^{+}$ is an element of the set $K_{g}$ of eigenmeasures as defined by Walters in [13]. And vice versa, any $\nu$ in $K_{g}$ corresponds, by extension, to a unique $g$-chain.

Proof. Suppose that we have $P^{*} \nu=\nu$. This is equivalent with $\mathcal{L}^{*}\left(\nu \circ T^{-1}\right)=\nu$. By interpreting the conditional probability $\nu\left(x_{0} \mid x_{1}, x_{2}, \ldots\right)$ as the transition probability $\nu\left(x^{(0)} \mid x^{(-1)}\right)$ from the Markov chain defined above, we reach the conclusion that

$$
\nu\left(x_{0} \mid x_{1}, x_{2}, \ldots\right)=g\left(x_{0}, x_{1}, \ldots\right) .
$$

Thus, if $P^{(n)^{*}} \nu=\nu$, we have for $0 \leq k \leq n-1$

$$
\nu\left(x_{k} \mid x_{k+1}, x_{k+2}, \ldots\right)=g\left(x_{k}, x_{k+1}, x_{k+2}, \ldots\right) .
$$

Since $K_{g}^{n}=P^{(n)^{*}} \mathcal{P} X^{+}$and $K_{g}=\bigcap_{n=1}^{\infty} K_{g}^{n}$, we have precisely (2.2) for all $k \geq 0$. Using measures in the nonempty set $K_{g}$ as initial distributions, we may define the full $g$-chain, by making a unique canonical extension (Neveu [9], p. 83 , the corollary), so that (2.2) is true for $k \leq-1$. Hence (2.2) holds for all $k \in \mathbb{Z}$.

From now on, we do not distinguish between a $g$-chain $\nu$ and its one-sided restriction $\nu \circ \Pi_{+}^{-1}$.

Lemma 2.4. The set $K_{g}$ is a non-empty convex subset with mutually singular extreme points.

Proof. See Theorem 2.11 and its proof in Walters [13].

We work under the assumption that $g>0$ and hence that any two $g$-chains be will locally a.c. on any of the algebras $\mathcal{F}_{a}^{b}$, where $a$ and $b$ are finite. Given two $g$-chains, $\nu, \tilde{\nu} \in K_{g}$, let

$$
\xi_{n}(x):=Z_{n}\left(x ; \tilde{\nu}, \nu,\left\{\mathcal{F}_{n}^{+}\right\}\right)
$$

Note that $\xi_{n}$ is the likelihood-ratio martingale and since it is a positive $L_{1}(\nu)$ bounded $\nu$-martingale, we know that it converges $\nu$-almost surely. We want to show that it is uniformly integrable, $U I$, with respect to $\nu$, which gives an $L_{1}$ density between $\tilde{\nu}$ and $\nu$ on $\mathcal{F}^{+}=\lim \mathcal{F}_{n}^{+}$. And thus, according to Lemma 2.4, a contradiction if $\nu$ and $\tilde{\nu}$ are chosen to be distinct and extremal in $K_{g}$. 
It was shown in [7] (p. 595) that the UI property of a likelihood-ratio martingale such as $\xi_{n}$ amounts to show that

$$
\lim _{K \rightarrow \infty} \sup _{n} \tilde{\nu}\left(\log \xi_{n}>K\right)=0 .
$$

To see (2.4), we note that, for a fixed $m$, a translation $m$ steps to the left of both the point and the measure gives that

$$
\xi_{m}(x)=Z_{m}\left(T^{m} x ; \tilde{\nu} \circ T^{-m}, \nu \circ T^{-m},\left\{\mathcal{F}_{m}^{-}\right\}\right) .
$$

and hence the law of $\xi_{m}$ under $\tilde{\nu}, \tilde{\nu} \circ \xi_{m}^{-1}$, equals the distribution $\left(\tilde{\nu} \circ T^{-m}\right) \circ \zeta_{m, m}^{-1}$ where $\left(\zeta_{m, n}(x): n \in \mathbb{Z}_{+}\right)$is the forward likelihood ratio martingale ( $m$ is fixed)

$$
\zeta_{m, n}(x)=Z_{n}\left(x ; \tilde{\nu} \circ T^{-m}, \nu \circ T^{-m},\left\{\mathcal{F}_{n}^{-}\right\}\right) .
$$

This means that we start with two extremal measures $\nu, \tilde{\nu} \in K_{g}$ translated $m$ times to the left, i.e., $\nu \circ T^{-m}$ and $\tilde{\nu} \circ T^{-m}$, which can be extended to well-defined $g$-chains and then we may go forward along $\mathcal{F}_{n}^{-}$.

Thus, it is enough to show the following lemma stating that the forward likelihood ratios are uniformly tight in a strong sense. The estimates have to be uniform in all $g$-chains, since our substitutions $\mu=\nu \circ T^{-m}$ and $\tilde{\mu}=\tilde{\nu} \circ T^{-m}$ are valid only for a fixed $m$.

Lemma 2.5. Assume that $\operatorname{var}_{n} g$ is square summable and that $g$ is bounded away from zero. For all $\epsilon>0$ there is a $K=K(\epsilon)$ such that

$$
\sup _{\tilde{\mu}, \mu} \sup _{n} \tilde{\mu}\left(\log Z_{n}\left(x ; \tilde{\mu}, \mu,\left\{\mathcal{F}_{n}^{-}\right\}\right)>K\right)<\epsilon,
$$

where $\tilde{\mu}$ and $\mu$ are chosen among all pairs of forward $g$-chains.

Proof. We adapt the part of the proof in [7], pp. 597-598. One could also adapt the more advanced theory of Shiryaev [11] and his co-authors, as was done in [8].

Given $\mu$ and $\tilde{\mu}$ write (with notation from [7]) $M_{n}(x)=Z_{n}\left(x ; \tilde{\mu}, \mu,\left\{\mathcal{F}_{n}^{-}\right\}\right.$). We show that $\log M_{n}$ has a Doob decomposition (see e.g. [14], pp. 120-121):

$$
\log M_{n}=A_{n}+\eta_{n}
$$

where $A_{n}$ is previsible with the uniform bound $A_{n} \leq C_{1} \sum\left(\operatorname{var}_{n} g\right)^{2}$, and moreover, $\eta_{n}$ is a $\tilde{\mu}$-martingale and uniformly bounded in $L_{2}(\tilde{\mu})$ with $\tilde{\mu}\left(\eta_{n}^{2}\right) \leq$ $C_{2} \sum\left(\operatorname{var}_{n} g\right)^{2}$. It is the uniformity of the estimates which makes it possible to conclude the strong formulation of (2.6). We define

$$
\begin{aligned}
P_{n}(x) & =\frac{\mu\left[x_{-n}, x_{-n+1}, \ldots, x_{-1}\right]}{\mu\left[x_{-n+1}, \ldots, x_{-1}\right]} \\
\tilde{P}_{n}(x) & =\frac{\tilde{\mu}\left[x_{-n}, x_{-n+1}, \ldots, x_{-1}\right]}{\tilde{\mu}\left[x_{-n+1}, \ldots, x_{-1}\right]}
\end{aligned}
$$


and note that $\left|\tilde{P}_{n}-P_{n}\right| \leq \operatorname{var}_{n} g$.

The proof then follows exactly as in [7], pp. 597-598.

\section{Open questions}

In this section we present two questions which the authors find interesting, as well as challenging, in the light of the present investigation.

3.1. Question 1. Does uniqueness of a $g$-measure imply

$$
\mathcal{L}^{n} f \rightarrow \int f d \mu ?
$$

We assume that we have finitely many symbols for the left shift map $T$ and a continuous and strictly positive $g$-function.

In this paper we used that a unique measure in $K_{g}$ (Walters notation), or equivalently, a unique $G$-measure (Brown and Dooley [2], Fan [4], and others), implies that $\mathcal{L}^{n} f(x) \rightarrow \int f d \mu$, uniformly in $x \in X^{+}$. It would be natural to ask that if $K_{g}$ only contains the $g$-measure, then is this the unique member also of $K_{g}$ (recall that a $g$-measure is always a member of $K_{g}$ )? Then it would follow that uniqueness of a $g$-measure (under the assumptions given in our question) implies convergence of the iterates of $\mathcal{L}$, without imposing stronger regularity on $g$ than continuity.

3.2. Question 2. Under square summability of variations of the $g$-function, what is the rate of convergence of $\mathcal{L}^{n} f(x)$ in the supremum-norm? Rates of convergence in the case of summable variations are available, see Pollicott [10].

Acknowledgements. The authors are grateful for the hospitality shown by the University of Warwick and in particular for the conversations with Thomas Jordan, Mark Pollicott and Peter Walters.

\section{REFERENCES}

[1] N. Berger, C. Hoffman and V. Sidoravicius, Nonuniqueness for specifications in $l^{2+\epsilon}$. Preprint available on www.arxiv.org (PR/0312344).

[2] G. Brown and A.H. Dooley, Odometer actions on $G$-measures, Ergodic Theory and Dynam. Systems 11 (1991), 279-307.

[3] A.H. Dooley and Ö. Stenflo, A criterion for uniqueness in $G$-measures and perfect sampling, Math. Proc. Camb. Philos. Soc. 140 (2006), 545-551.

[4] A.H. Fan, On uniqueness of $G$-measures and $g$-measures, Studia Math. 119 (1996), no. 3, 255-269.

[5] P. Hulse, An example of non-unique g-measures, Ergodic Theory Dynam. Systems 26 (2006), 439-445.

[6] M. Keane, Strongly Mixing g-Measures, Invent. Math. 16 (1972), 309-324. 
[7] A. Johansson and A. Öberg, Square summability of variations of $g$-functions and uniqueness of $g$-measures, Math. Res. Lett. 10 (2003), no. 5-6, 587-601.

[8] A. Johansson, A. Öberg and M. Pollicott, Countable state shifts and uniqueness of $g$-measures, to appear in Amer. J. Math. Preprint available at http://www.math.uu.se/ anders/forskning.html.

[9] J. Neveu, Matematical Foundations of the Calculus of Probability, Holden-Day, 1965.

[10] M. Pollicott, Rates of mixing for potentials of summable variation, Trans. Amer. Math. Soc. 352 (2000), 843-853.

[11] A.N. Shiryaev, Absolute Continuity and Singularity of Probability Measures in Functional Spaces, Proceedings of the International Congress of Mathematicians, Helsinki 1978, pp. 209-225.

[12] P. Walters, Ruelle's operator theorem and g-measures, Trans. Amer. Math. Soc. 214 (1975), 375-387.

[13] P. Walters, Convergence of the Ruelle operator for a function satisfying Bowen's condition, Trans. Amer. Math. Soc. 353 (2001), no. 1, 327-347.

[14] D. Williams, Probability with Martingales, Cambridge University Press 1991.

Anders Johansson, Division of Mathematics and Statistics, University of Gävle, SE-801 76 GäVLE, SWEDEN

E-mail address: ajj@hig.se

Anders Öberg, Department of Mathematics, Uppsala University, P.O. Box 480, SE-751 06 Uppsala, Sweden

E-mail address: anders@math.uu.se 